P.R. Mohapatra and D.T. Hari also point out that we do not consider the benefits of using intermittent therapy. Again, the primary objective of our study was to consider gains that could be made by switching to a WHO-supported standardised regimen, one in which daily therapy is recommended wherever feasible [2].

\section{O. Oxlade and D. Menzies}

Montreal Chest Institute, Respiratory Epidemiology Unit, Montreal, Quebec, Canada.

Correspondence: D. Menzies, Respiratory Epidemiology Unit, Montreal Chest Institute, 3650 St Urbain, Room K1.24,
Montreal, Quebec, Canada, H2X 2P4. E-mail: dick.menzies@ mcgill.ca

Statement of Interest: None declared

\section{REFERENCES}

1 Oxlade $\mathrm{O}$, Schwartzman $\mathrm{K}$, Pai M, et al. Predicting outcomes and drug resistance with standardised treatment of active tuberculosis. Eur Respir J 2010; 36: 870-877.

2 World Health Organization. Treatment of Tuberculosis Guidelines. 4th Edn. Report No. WHO/HTM/TB/2009.420. Geneva, World Health Organization, 2010.

\title{
Estimated population prevalence of obstructive sleep apnoea in a community of German third graders
}

\section{To the Editors:}

We read with interest the article by URSCHITZ et al. [1] which estimated the population prevalence of obstructive sleep apnoea (OSA) in an urban community of German third graders. This article highlights the fact that OSA is one of the most common chronic respiratory diseases in childhood and requires more attention from paediatric health services and clinicians. However, we are concerned that the methods employed in the study to diagnose OSA were suboptimal.

The authors correctly state that full sleep laboratory based polysomnography is the gold standard for the diagnosis of OSA in children. However, for the purposes of their study, abbreviated home polysomnography (HPSG) without electroencephalography was utilised for a final diagnosis of OSA. This was not referenced to the gold standard in this study.

The most recent American Academy of Sleep Medicine manual on scoring of sleep and sleep-related events emphasises the need to record and score not only respiratory events, but also arousals throughout a polysomnography [2]. The manual states that if hypopnoeas are to be scored (and form part of the apnoea/ hypopnoea index), they must be followed by either arousal or desaturation. The abbreviated HPSG used in this study does not have the ability to detect respiratory events that lead to arousal. On this basis, we feel that the authors may have underestimated the prevalence of OSA in their study population.

\section{Finn and P. McNally}

Dept of Respiratory Medicine, Our Lady's Children's Hospital, Crumlin, Dublin 12, Ireland.

Correspondence: P. McNally, Dept of Respiratory Medicine, Our Lady's Children's Hospital, Crumlin, Dublin 12, Ireland. E-mail: paul.mcnally@olchc.ie
Statement of Interest: None declared.

\section{REFERENCES}

1 Urschitz MS, Brockmann PE, Schauld M, et al. Population prevalence of obstructive sleep apnoea in a community of German third graders. Eur Respir J 2010; 36: 556-568.

2 Iber C., Ancoli-Israel S., Chesson AL., et al., eds. The AASM Manual for the Scoring of Sleep and Associated Events: Rules, Terminology and Technical Specifications. Westchester, American Academy of Sleep Medicine, 2007.

DOI: $10.1183 / 09031936.00185610$

From the authors:

We thank D. Finn and P. McNally for their comments on our recent publication in the European Respiratory Journal [1].

We agree that full sleep laboratory-based polysomnography (PSG) including electroencephalography (EEG) may have a higher sensitivity in detecting obstructive sleep apnoea (OSA) in children, and that its use may have led to a higher estimate for the prevalence of OSA in our study. However, our study was designed in 2000, long before the relevant guideline of the American Academy of Sleep Medicine (AASM) was published [2]. At that time, there was widespread debate about the way in which hypopnoea and arousals should be scored and about the significance of these events in children. Many authors used definitions for hypopnoea that were based solely on a reduction in airflow or on both a reduction in airflow and desaturation. In a study by MCNAMARA et al. [3], only 38-39\% of respiratory events (including hypopnoea) were followed by cortical arousal, while all hypopnoeas were followed by a desaturation of $\geqslant 4 \%$. In addition, the occurrence of a cortical arousal following respiratory events depended on age. In contrast to the current AASM guideline, the minimal required duration for a cortical 